\title{
Die Blechtrommel in der finnischen Neuübersetzung von Oili Suominen. Zur Vernetzung von Verlag, Übersetzung und Rezeption
}

\author{
Liisa Laukkanen
}

\begin{abstract}
This article deals with the brief revival of interest in Grass's Die Blechtrommel when the second Finnish translation appeared in 2009 to commemorate the fiftieth anniversary of the novel's first publication. The new translation was undertaken on the initiative of the translator Oili Suominen who can be considered the Finnish translator of Grass's works. The translation is accompanied by the translator's own explanatory notes and an afterword by the writer, critic and poet Jukka Koskelainen. In this paper the role of the translator along with the publisher and the critics as active participants in the public reception process is discussed.

The article also discusses how the publisher's publicity material influences the reception of the work as an accepted classic. It also reveals how the reviewers absorb and disseminate the material provided in the afterword. Comparing reviews in the print media versus readers' blogs reveals a major difference. The print media concentrate on the qualities of the new translation, whereas the blogs are more interested in the book itself. The blogs may well be motivated by the publicity surrounding the new translation, but they do not make any use of it and do not mention the translator at all. Although the new translation sold quickly, the initial edition was not followed up with reprints. Grass clearly does not have a broad readership in Finland and at time of writing his books are mostly out of print.
\end{abstract}

\section{Einleitung}

In einer Situation, in der sich die Welt immer mehr multikulturell gestaltet, spielen Übersetzungen eine wichtige Rolle für die interkulturelle Interaktion. ${ }^{1}$ Literarische Werke aus einer anderen Sprache werden in einem fremden Kulturkontext in der Regel in Form von Übersetzungen gelesen. Es sind dann

\footnotetext{
1 Vgl. Launis (2012) 27.

(C) LIISA LAUKKANEN, 2016 | DOI 10.1163/9789004291898_012

This is an open access chapter distributed under the terms of the CC-BY-NC License.
} 
auch die Übersetzungen, die rezipiert werden, was zu unterschiedlich gewichteten Rezensionen der Werke führen kann: Man analysiert das Werk so, als sei es ein Originalwerk oder hebt die Übersetzung in den Mittelpunkt der Rezension, oder aber macht keinen Unterschied zwischen den beiden Herangehensweisen. Wie die Rezeption sich auch immer gestaltet, so spielt jedoch der zeitliche Aspekt eine besondere Rolle beim Leser und Rezensenten: Jede Generation kann neue Gesichtspunkte an einem Werk der Literatur - ob in der Originalsprache oder in Übersetzungen - entdecken, was die ,Polyinterpretabilität ${ }^{2}$ eines Kunstwerks erhöht. Wie sieht die Polyinterpretabilität in den Rezensionen derjenigen Werke aus, die bereits übersetzt worden sind und sich einer Neuübersetzung erfreuen? Auf diese Frage soll im Folgenden anhand der Rezeption der zweiten finnischen Übersetzung der Blechtrommel von Günter Grass eingegangen werden, die 2009, zehn Jahre nach der Verleihung des Nobelpreises an Grass und zugleich 50 Jahre nach dem Erscheinen des Originalwerks, in der Übersetzung von Oili Suominen erschien. Die Übersetzung ist mit Erläuterungen der Übersetzerin und einem Nachwort des Schriftstellers, Literaturkritikers und Essayisten Jukka Koskelainen versehen.

Nicht nur die Rezensenten des Werks, sondern auch der Verlag und die Übersetzerin werden hier als aktive Akteure im Prozess der Literaturvermittlung verstanden. Von dieser Prämisse ausgehend wird zuerst auf den Verlag Otava und seine Bedeutung für die Rezeption eingegangen, was eng mit der rezeptionssteuernden Funktion der Übersetzerin Suominen im Vermittlerprozess zusammenhängt. Die daran anschließenden Bemerkungen und Überlegungen zur Problematik einer Neuübersetzung leiten die nähere Betrachtung der Rezeptionsdokumente ein, die aus Epitexten und insgesamt neun Rezensionen zum Buch bestehen.

\section{Der Verlag Otava}

Sämtliche finnischsprachigen Ausgaben der Blechtrommel sind im Verlag Otava erschienen, der zu den ältesten und größten Verlagshäusern Finnlands gehört. In der finnischen Verlagslandschaft gilt der Verlag seit seiner Gründung als ein liberales Haus, das noch heute als seine Mission "die Bildung der Nation durch Schaffen von Erlebnissen und Erkenntnissen“ ${ }^{3}$ proklamiert. ${ }^{4}$ Dies

\footnotetext{
2 Pöckl (2011) 46.

3 Otava (2014).

4 Sämtliche Übersetzungen von L.L.
} 
geschehe in einem „lebendigen Umgang mit der übrigen Welt“, 5 was sich konkret in der Veröffentlichung der Werke von ,Ikonen ${ }^{6} 6$ wie Nobelpreisträgern zeigt. Dies muss der ideologische Leitfaden bereits auf der Frankfurter Buchmesse um die Jahrzehntwende 1950/196o gewesen sein, als Otava im Auftrag von vier nordischen Verlagen in einer halben Stunde die Rechte für die Übersetzung des Romans verhandeln konnte. ${ }^{7}$ Der Bildungsauftrag des Verlags hat sich in der letzten Zeit neben Belletristik insbesondere um das Verlegen von Lehrmaterial, Wörterbüchern und Lexika erweitert.

Wenn Otava auf der einen Seite seinen Bildungsauftrag hervorhebt, so weist er auf der anderen Seite gleichzeitig auf die kommerzielle Bedeutung des Buchs für den Verlag hin: Das Buch sei bereits seit 1950/1960 „immer deutlicher und unausweichlicher ein Gegenstand, eine ,Ware‘, die Geld ins Haus bringen soll. ${ }^{8}$ Heute, gut $5^{0}$ Jahre später, zeigt sich, dass bzgl. der Blechtrommel vom Bildungsauftrag nichts mehr zu spüren ist. Die Ware Blechtrommel ist nicht mehr auf dem Markt: Weder Die Blechtrommel noch weitere Werke von Günter Grass noch Günter Grass selbst als Autor sind auf den aktuellen Internetseiten des Verlags zu finden. ${ }^{9}$ Die Blechtrommel ist ausverkauft und in das Angebot der Antiquariate gerutscht bzw. nur in den öffentlichen Bibliotheken vorhanden, ob nun in erster oder in zweiter Übersetzung. Wenn keine Neuauflagen vorhanden sind, so heißt das zugleich, dass das Werk nur noch vereinzelt rezipiert wird, und zwar in der Auflage, die dem Rezipienten gerade zur Verfügung steht.

Die Rezeption kann mit den von der Werbeabteilung des Verlags gestalteten Werbetexten gesteuert werden. In der Verlagsankündigung vom März 2009 bezeichnet Otava die Übersetzung von Suominen als ausgezeichnet. Das Buch wird als ein Klassiker, als einer der wichtigsten Romane der europäischen Literatur des 20. Jahrhunderts charakterisiert, der zum 50. Jubiläumsjahr in neun Sprachen, inklusive Finnisch, neu übersetzt wurde. Das Jubiläum ist einer der Gründe, den z. B. Pöckl ${ }^{10}$ erwähnt, wenn es darum geht, für das 'Warum' einer Neuübersetzung eine Antwort zu finden. Neben einer Kurzinhaltsangabe wird in der Verlagsankündigung das Nachwort von Koskelainen erwähnt.

\footnotetext{
$5 \quad$ Otava (2014).

6 Otava (2014).

$7 \quad$ Lassila (1990) 211.

$8 \quad$ Lassila (1990) 378.

9 Nach dem Tod des Autors ist inzwischen eine neue Taschenbuchausgabe der Blechtrommel erschienen. Auch sie ist nur bei gezielter Suche auf der Homepage des Verlags auffindbar [Anm. der Redaktion].

Pöckl (2004) 201.
} 
Zudem wird aus der Rezension von Pentti Saarikoski zur ersten Übersetzung Folgendes übernommen: „Die Blechtrommel ist grotesk, wild, frech; unmöglich und amüsant - obszön und lebensbejahend. Es ist unnötig darüber mehr zu sagen als das im Sinne von Oskar: Nehmt und esst!"11 Die intertextuellen Verweise auf die Tote(n) Seelen von Gogol und auf den Roman Gargantua von Rabelais in Saarikoskis Rezension wurden dagegen nicht übernommen. ${ }^{12}$ Die Werbung beschränkt sich auf diese Ankündigung, so dass der Werbeaufwand des Verlags recht bescheiden ausfällt.

\section{Die Neuübersetzung}

Die Rezeption der Neuübersetzung der Blechtrommel (2009) in Finnland geht besonders in den Printmedien sehr stark auf die übersetzerische Leistung von Suominen ein. Der Schriftsteller und Übersetzer Felix Philipp Ingold bezeichnet eine Neuübersetzung als eine ,Nachübersetzung ${ }^{13}$. Bei den Nachübersetzungen kann es darum gehen, eine bereits vorliegende Übersetzung zu revidieren, also philologisch auf Fehler oder Auslassungen im Text zu überprüfen. Zudem kann ein Bedürfnis einer Modernisierung der Textvorlage in einem zeitlich anderen Kontext mit anderen Lektüreerwartungen bestehen. ${ }^{14}$ Bei der Neuübersetzung der Blechtrommel treffen aus der Sicht der Übersetzerin und des Verlags beide Aspekte zusammen.

Wie der Verlag in seiner Werbeanzeige betonte, sei Die Blechtrommel ein Klassiker, und es sind vor allem Klassiker, die neu übersetzt werden. Als solche können laut Stefan Neuhaus „als besonders gelungen beurteilte, paradigmatische Werke (verstanden werden), deren Leistung in einer Epoche bzw. für die innovationsgesteuerte Entwicklung der Literatur nicht mehr hinterfragt wird“ ". Wenn ein solches Werk übersetzt wird, dann ändert sich auch sein Status: Es wird zum Teil der Weltliteratur, ${ }^{16}$ wie auch im Nekrolog auf Grass in Helsingin Sanomat festgestellt wurde. ${ }^{17}$ Problematisch werden die Begriffe ,Klassiker und ,Weltliteratur' allerdings dann, wenn das übersetzte Werk relativ schnell vergriffen ist und nicht neu aufgelegt wird. Es stellt sich die Frage, wie und wie

\footnotetext{
$11 \quad$ Saarikoski (1961) 258.

12 Saarikoski (1961) 258.

13 Ingold (2007).

14 Vgl. auch Pöckl (2004).

15 Neuhaus (2009) 69.

16 Oittinen (2000) 271.

17 Majander, Petäjä (2015).
} 
lange unter solchen Umständen der Klassikerstatus aufrecht erhalten bleiben kann und in welchen Kontexten dieser Status wieder re-aktualisiert wird.

In der Forschung wird die These aufgestellt, dass neue Übersetzungen dem Ausgangstext ,treuer' sein können, da die erste Übersetzung den Text dem Zielpublikum bereits vorstellt, sowohl was das Thema als auch was die kulturellen Begebenheiten betrifft. ${ }^{18}$ In der Neuübersetzung hat der Übersetzer die Möglichkeit, die Vorgängerversion als Basis in Anspruch zu nehmen und darauf seine Übersetzung zu gestalten. Sprachliche und kulturelle Elemente der Vorgängerversion können geändert oder unverändert übernommen werden. Ingold deutet in diesem Zusammenhang auch auf die ,Konkurrenz der Stimmen' hin, da der Übersetzer seinerseits die Berechtigung einer Neuübersetzung durch seine Arbeit ausweisen muss. ${ }^{19}$

Die finnische Neuübersetzung der Blechtrommel ist ausdrücklich auf die Initiative der Übersetzerin Suominen entstanden, die dem Werk ,magischen Realismus ${ }^{20}$ zuspricht. Sie hat zuerst Kontakt mit dem Verlag Otava aufgenommen und sich anschließend darüber mit Günter Grass unterhalten, wie Grass in einem Interview mit dem finnischen Redakteur Teppo Tiilikainen betont. ${ }^{21}$ Eine neue Blechtrommel-Übersetzung ist aus der Sicht der Übersetzerin deshalb aktuell geworden, weil die Sprache sich verändert hat und die Übersetzung diesbezüglich etwas veraltet sei. ${ }^{22}$ Dies wiederum kann u. a. darauf zurückgeführt werden, dass Hilfsmittel zur Zeit der ersten Übersetzung mangelhaft waren. So ist z. B. die Übersetzung der zeitgebundenen Fachterminologie im Buch problematisch gewesen, da nur einbändige Wörterbücher zur Verfügung gestanden hatten. ${ }^{23}$

Der Bedarf an einer Neuübersetzung wurde zusätzlich durch die veränderte Literaturszene erhöht. Ende der 5oer Jahre, also zur Zeit der ersten Übersetzung, hatte man sich noch nicht an den grotesken Stil von Grass gewöhnt, denn keiner hatte bis dahin so geschrieben wie Grass, so die Übersetzerin Suominen, die als die Grass-Übersetzerin in Finnland gilt. ${ }^{24}$ Sie stellt weiter fest, dass die ersten Übersetzungen überhaupt ,tastend' waren, da der Text so ,reich` und

\footnotetext{
18 Tiittula (2013) $145^{-146 .}$

19 Ingold (2007).

20 Ahola (2015).

21 Tiilikainen (2009).

$22 \quad$ Toivanen (2006) 51.

23 Lähteenmäki (2009) 5 .

24 Oili Suominen hat insgesamt 13 Bücher von Grass übersetzt: Hundejahre, Der Butt, Das Treffen in Telgte, Kopfgeburten, Zunge zeigen, Die Rättin, Unkenrufe, Ein weites Feld, Mein Jahrhundert, Im Krebsgang, Beim Häuten der Zwiebel und Die Box. Ihre Übersetzung von Grimms Wörter ist im Herbst 2015 erschienen.
} 
,ausschweifend' ist. ${ }^{25}$ Grass wiederum betont in dem Interview eher den ,erweiterten Realismus ${ }^{26}$ in seinem Werk und geht davon aus, dass die ersten Übersetzungen in andere Sprachen in viel zu großer Eile angefertigt wurden. Szenen wurden ausgelassen und die Übersetzungen seien sehr fehlerhaft. Laut Grass ist dies darauf zurückzuführen, dass er ,viele mundartliche Ausdrücke verwendet ${ }^{\prime}, 27$ die in andere Sprachen fehler- und mangelhaft übertragen wurden. Folglich unterscheiden sich die finnischen Übersetzungen, ziemlich stark ${ }^{\star}$ von einander. ${ }^{28}$

Als Schlussfolgerung der Diskussion über die Notwendigkeit einer Neuübersetzung konstatiert Suominen Folgendes: „Warum bräuchte man übrigens keine neue Übersetzung? Auch Schauspiele, Opern, Gedichte und Filme haben mehrere Aufführungen. Keiner fragt mehr, warum Tuntematon sotilas ${ }^{29}$ zweimal verfilmt worden ist und mindestens genauso viele Theateraufführungen erfahren hat. “30

Der Übersetzer ist immer auch ein Rezipient, da die Übersetzungliterarischer Texte Bestandteil der Rezeption eines Textes oder Werkes ist. ${ }^{31}$ Übersetzungen dokumentieren den Sprachzustand der jeweiligen Zeit sowie das vorhandene fachliche und kulturelle Vorwissen, d. h. den Erwartungshorizont des Übersetzers. ${ }^{32}$ Die Erweiterung des Erwartungshorizonts kann anhand des Übersetzungs- und Vorbereitungsprozesses von Suominen auch konkret aufgezeigt werden: Nach ihren eigenen Worten hat sie das Werk mindestens fünf Mal vor der Übersetzung gelesen, sich Auszüge aus dem von Grass vorgelesenen Werk angehört und während der Übersetzung das Werk vier bis fünf Mal durchgelesen. Zudem hatte sie am Seminar mit Grass und weiteren Übersetzern teilgenommen, um Erläuterungen zum Roman von Grass selbst zu bekommen. Sie hatte auch die Theateraufführung vom Q-Theater besucht, ${ }^{33}$ die in der Presse sehr positiv rezipiert wurde. ${ }^{34}$

Was die praktische Durchführung der Übersetzung betrifft, so bestätigt Suominen, dass die Arbeit eine Herausforderung war: „Die Blechtrommel hat

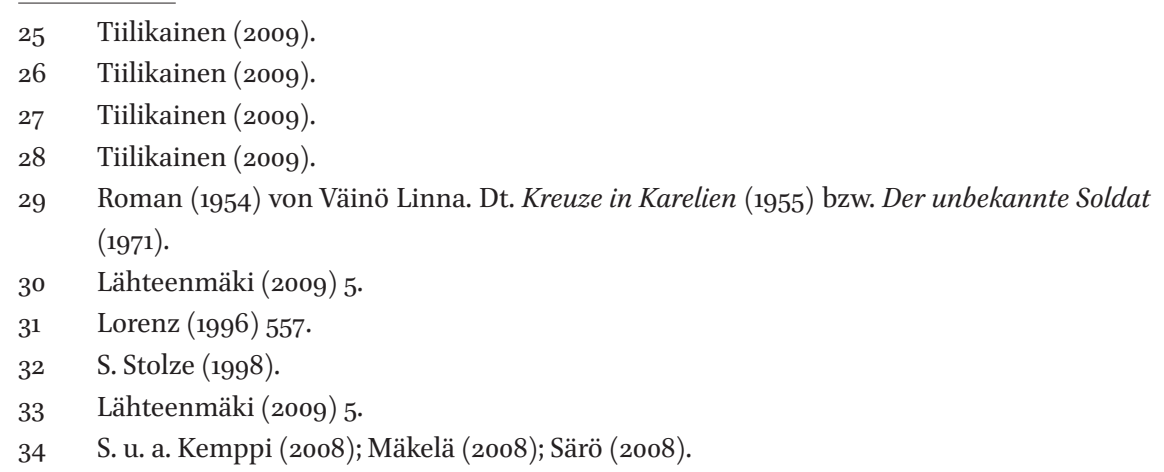


mehrere Ebenen und ist unerschöpflich, das Buch kann auf viele Weisen gelesen und interpretiert werden. Es ist ein Buch für alle Sinne. Es ist sehr visuell wie alle Werke von Grass - er ist ja auch Vertreter der bildenden Kunst, und seine Werke entstehen zuerst als Bilder auf dem Papier.“35 Sprachlich ist Die Blechtrommel anspruchsvoll, denn im Text gibt es viele Allusionen und unterschiedliche Verweise auf die Wirklichkeit außerhalb des Buchs. Diese außertextuellen Verweise werden häufig nicht übersetzt, sie müssen lediglich verstanden und geklärt werden, weil man sie beim Übersetzen mitberücksichtigen muss, so Suominen weiter. ${ }^{36}$ Zudem verwendet Grass in seinem Buch unterschiedliche Stilregister, und es werden fast alle rhetorischen Mittel in Anspruch genommen. Auch die Satzstruktur ist manchmal sehr kompliziert. Ebenfalls ist die Welt der Laute, verbunden mit vielen Gerüchen und Düften, ein für die Blechtrommel typisches Merkmal. ${ }^{37}$

Die von Suominen angesprochene Mehrdeutigkeit kann u. a. am Vergleich der beiden Übersetzungen festgehalten werden. Als Beispiel für einen Übersetzungsfehler sei das homonyme Substantiv „Rock“38 erwähnt, das in der Übersetzung von Peromies ${ }^{39}$ als ein Frauenrock (engl. skirt) wiedergegeben wird, während Suominen ${ }^{40}$ den Kontext des Lexems mitberücksichtigt und die der Situation passende Übertragung, nämlich als ,Uniform', verwendet. ${ }^{41}$ Weitere Beispiele dieser und ähnlicher Art sind u. a. in der Analyse von Liisa Tiittula zu finden. Sie hat die beiden Übersetzungen von 1961 und 2009 exemplarisch auf Verständlichkeit, Erläuterungen, Satzzeichen und Übertragung der Mündlichkeit untersucht und festgestellt, dass bei der Übertragung der Mündlichkeit die Neuübersetzung dem Original , treuer ${ }^{42}$ sei. Ihr Fazit ist allerdings keine Überraschung: „Mal folgt die eine, mal die andere Übersetzung dem Ausgangstext genauer (...).“43 


\section{Peritexte der Ausgabe 2009}

Die Blechtrommel-Ausgabe von 2009 wurde vom Komitee der Buchkunst Finnlands zum schönsten Buch des Jahres 2009 in der Kategorie Belletristik auserkoren. In der Begründung heißt es u. a.: „Dieser Buchklassiker hat neue beeindruckende Kleider bekommen. Die ungewöhnliche aber stilvolle Farbgebung, der wertvolle Einband und die mutige Namenstypographie sowie die Zeichnung von Grass schaffen eine ausgewogene Ganzheit“. 44

Schaut man sich die im Umfeld des Textes liegenden Peritexte genauer an, so gleitet der Blick zuerst auf das Buchcover (ohne Schutzumschlag) und die Rückseite des Buchs. Das Buchcover mit dem Namen des Autors, Titel des Werks, Namen des Übersetzers und dem Verlag fällt wegen der oben erwähnten, vom Buchkunst-Komitee gelobten Aufmachung auf. Für alle finnischsprachigen Ausgaben erweist sich die Abbildung der Blechtrommel als gemeinsames semiotisches Zeichen. Auf der Rückseite wird das Leben von Oskar Matzerath zusammengefasst. Des Weiteren wird der Nobel-Preis für Günter Grass erwähnt sowie auf die Erscheinung des Originalwerks vor $5^{0}$ Jahren und auf das Nachwort von Koskelainen hingewiesen. Zum Schluss dieses verlegerischen Informations- und Werbetextes folgen wieder Auszüge aus der Rezension von Saarikoski, ${ }^{45}$ die z. T. auch in der Verlagsankündigung zu finden sind. Im Werk gibt es noch zwei vom Autor Grass verfasste Peritexte: eine Widmung an Anna Grass sowie einen sogenannten Fiktionsvertrag, ${ }^{46}$ eine Beteuerung der Fiktionalität: „Personen und Handlung des Buches sind frei erfunden.Jede Ähnlichkeit mit einer lebenden oder verstorbenen Person ist nur zufällig." Eine solche, eher in Filmen vorzufindende autonome Formulierung spekuliert in einem fiktionalen Text gerade darauf, dass der Leser sich auf die Suche nach solchen Ähnlichkeiten macht. Folglich kann es sein, dass der Text „in entscheidenden Teilen nicht fiktional gelesen“47 wird. Diese Beteuerung wurde von der Rezensentin Tarja Pulli als „amüsant“ bezeichnet. ${ }^{48}$

Das Nachwort von Koskelainen hat zum Einen die Funktion, das Werk zu ergänzen oder zu erläutern. Es wendet sich an den tatsächlichen Leser ${ }^{49}$, also an einen Leser, der das Werk bereits gelesen hat. Zum Anderen lenkt es deutlich die literaturkritische Rezeption, da die Rezensenten ihre Texte nicht

\footnotetext{
44 Suomen kirjataiteen komitea (2009).

45 Saarikoski (1961).

46 Genette (2001) 209.

47 Bode (2005) 11.

48 Pulli (2009).

49 Genette (2001) 229.
} 
selten anhand der Informationen im Nachwort formulieren. Das zehn Seiten umfassende Nachwort mit dem Titel „Von unten sieht man besser - die umgekehrte Perspektive von Günter Grass" ${ }^{40}$ beginnt mit der Hervorhebung dessen, was Günter Grass mit diesem Werk und der Danziger Trilogie insgesamt seiner Heimatstadt Danzig/Gdansk habe zuteilwerden lassen: Einerseits hat er der Stadt einen unvergesslichen Platz in der Weltliteratur gesichert, andererseits ein literarisches Monument für die Stadt gesetzt. Nicht unerwartet stellt Koskelainen einen Vergleich mit James Joyce und Dublin dar. Eher unerwartet werden dagegen anschließend fast zwei Seiten lang die Orte in Danzig, die im fiktiven Roman erwähnt werden, mit dem Besuch des finnischen Schriftstellers Olavi Paavolainen ${ }^{51}$ in Danzig in Verbindung gesetzt. ${ }^{52}$ Mit seinen Beobachtungen und Zitaten können laut Koskelainen die finnischsprachigen Leser in die zentralen Ereignisse in der Blechtrommel eingeführt werden. Die Rezensionen zeigen, dass sich diese Leselenkung als erfolgreich erweist.

Das Nachwort hebt weiter die Person Grass und den sozialgeschichtlichschriftstellerischen Kontext des Autors hervor: Biographische Angaben, auch die Mitgliedschaft in der Waffen-ss, der Einfluss anderer Schriftsteller auf die Produktion von Grass und insbesondere auf die Entstehung der Blechtrommel, die Bedeutung der Gruppe 47, die Buchkritik von Marcel Reich-Ranicki sowie die Kritik der katholischen Kirche in Deutschland finden hier Platz. Weitere außertextuelle Beobachtungen schließen auch die Diskussion über Grass und den ss-Skandal in den deutschen Medien mit ein, was nach Koskelainen auf das Bild eines „öffentlichen Intellektuellen, scharfen politischen Redners einen Fleck hinterlassen “53 habe. Die Werkanalyse konzentriert sich auf die Hauptfigur und auf den Stil im Roman. Seine Schlussfolgerungen formuliert Koskelainen in rhetorischen Fragen folgendermaßen: „Ist das Thema in der Blechtrommel und allgemein in den Werken von Grass letztendlich nicht dasjenige, dass die Welt ein widerspruchsvoller Ort und kein Mensch makellos sei? Dass Ideale wenig Gewicht haben, man allerdings im Tumult des Lebens auch etwas lernen kann, wenn man nicht im Bann der Ideale stecken bleibt. “54

Im letzten Peritext, in den von der Übersetzerin zusammengestellten Erläuterungen, werden folgende sieben Begriffe, die mit dem Ersten und

$50 \quad$ Grass (2009) 699-709.

51 Paavolainen (1903-1964) war in vieler Hinsicht „ein eigenartiges Phänomen im finnischen Kulturleben“ (Kansallisbiografia 2014). Er wird als Modernist bezeichnet, der aus kulturkritischer und -philosophischer Perspektive kulturelle, weltanschauliche und politische Ereignisse seiner Zeit beobachtete und über diese berichtete.

53 Grass (2009) 709.

54 Grass (2009) 709. 
Zweiten Weltkrieg zusammenhängen, erklärt: Harmannsweilerkopf in den Vogesen, Eintopfsonntag, PX (Christus-Monogramm), Kyffhäuserbund, ozN (Obóz Zjednoczenia Narodowego) sowie Beutedeutscher und Volkssturm. Mit diesen Erläuterungen soll Unbestimmtheitsstellen vorgebeugt werden, die evtl. bei den finnischsprachigen Lesern entstehen können. Die Erläuterungen bleiben in den Rezensionen allerdings unbeachtet.

\section{Rezeptionsdokumente als Epitexte}

Unter den dem Werk räumlich entfernteren Texten, den Epitexten, konnte folgendes Material ausfindig gemacht werden: Ein Artikel mit der Überschrift „Hintergrund“, drei Rezensionen in Printmedien sowie sechs Beiträge im Internet. Die Rezensionen sind in der größten finnischen Tageszeitung Helsingin Sanomat (2), in der Literaturzeitschrift Parnasso (1), im elektronischen Rezensionsorgan kiiltomato (1), im Blog der Frauenzeitschrift Olivia sowie in den Blogs Kemppinen, Tuumailua, Orapihlaja und Jokken kirjanurkka erschienen. Alle Verfasser der Printmedien-Artikel, die Verfasserin des OliviaBlogs sowie der Blogger Kemppinen bezeichnen sich als Redakteure oder Literaturkritiker. Die weiteren Blogger im Internet können als Buchliebhaber charakterisiert werden.

Der Rezension des Kulturredakteurs, Literaturkritikers und Literaturbloggers Hannu Marttila in Helsingin Sanomat (5.4.2009) geht am 4.4.20og sein Prätext mit der Überschrift „Hintergrund“ im digitalen Forum der Zeitung voraus. Im Prätext macht er den Leser auf den Unterschied zwischen dem Geburtsort „Danzig von Grass“ und dem literarischen „Gdansk in der Blechtrommel“ aufmerksam. ${ }^{55}$ Es geht um den Inhalt des Buchs nur insofern, als die geschichtliche Kontextualisierung und lokale Platzierung der Orte in Danzig, die auch im Roman zu finden sind, behandelt werden. Dass hier das Nachwort von Koskelainen mit den Hinweisen auf die Reise von Olavi Paavolainen den Rezensenten zu den Erläuterungen angeregt hat, wird im Beitrag zwar nicht explizit verbalisiert, kann jedoch nicht ausgeschlossen werden. Marttila weist den Leser deutlich darauf hin, dass die realen Orte nicht die Orte des fiktionalen Textes seien, sondern - wie Neuhaus ${ }^{56}$ schreibt -, der fiktionale Text nur mit der importierten Fremdreferenz real existierender Orte spielt.

Dies wird in Marttilas eigentlicher Rezension vom 5.4.2009 noch einmal besonders hervorgehoben. Diese erschien in der Printversion von Helsingin

55 Marttila (2009a).

$56 \quad$ Neuhaus 2009) 64f. 
Sanomat mit der Überschrift „Das Jesuskind des dunklen Jahrhunderts. Die Trommel von Oskar Matzerath schlägt in der neuen Übersetzung der Blechtrommel deutlicher"57. Marttila stellt seine subjektive Leseerfahrung an den Anfang, indem er schreibt, dass er neidisch auf diejenigen sei, die die Blechtrommel nun zum ersten Mal lesen dürften. Hiermit deutet er - wie bereits in der Überschrift seiner Rezension - auf die ,deutlichere' Neuübersetzung hin, auch wenn sie sich nicht „dramatisch“58 von der ersten unterscheidet: Sie „fließt“ einfach, „erfahrener und selbstsicherer als früher“..59 Im Mittelpunkt der weiteren Ausführungen steht zum einen der Vergleich der Übersetzungen vor dem Hintergrund der jeweiligen historisch-gesellschaftlichen Lage sowie der Arbeitssituation der beiden Übersetzer, in der diese ihre Übersetzungen angefertigt haben. Suominen habe in ihrer Übersetzung aufgrund der zeitlichen Distanz den historischen Kontext und den Stil von Grass besser mitberücksichtigen und auch die von ihr erwähnten internationalen Übersetzer-Werkstätte besuchen können, von denen der andere, der Verfasser der ersten Übersetzung Peromies, nicht einmal habe träumen können. Die Neuübersetzung erfülle die wichtigste Aufgabe einer Übersetzung, indem sie „das Erzählen des Schriftstellers unbemerkt vermittelt und unterstützt“. ${ }^{60}$ Der zweite Punkt, auf den der Rezensent eingeht, ist hermeneutisch und betrifft sein subjektives Werk-Verstehen: Er hatte beim Lesen der ersten Übersetzung im Jahr 1961 sein Augenmerk vor allem auf die Beschreibung der Zeit vor dem Krieg und während des Kriegs sowie auf dieses „heiße Thema in der europäischen und auch in der finnischen Literatur der sechziger Jahre" gerichtet. ${ }^{61}$ Beim Lesen der Neuübersetzung hat er nun das Jesus-Thema entdeckt - Oskar als Antichristus, der nicht vereint, sühnt, erlöst oder verzeiht. Wenn Oskar auf die Trommel schlägt, fängt das Publikum, die Nation, wieder an, sich zu erinnern - an all das, was sie bewusst und selektiert hat vergessen wollen.

Wenn bei Marttila vor allem die Übersetzungsthematik im Vordergrund steht, so enthält sich die Rezensentin Pulli62 in der Literaturzeitschrift Parnasso eines Vergleichs zwischen den beiden Übersetzungen. Sie stellt dazu lediglich fest, dass „die Sprache mit ihren sämtlichen Konnotationen einen Moment

\footnotetext{
57 Marttila (2009b).

$5^{8}$ Marttila (2009b).

59 Marttila (2009b).

60 Marttila (2009b).

61 Marttila (2009b), vgl. Parry in diesem Band.

62 Pulli (2009).
} 
lang“ lebt und „die Sprache von Suominen unaufhaltsam wie ein Frühlingsstrom"63 fließt.

Die Blechtrommel versteht sie als einen Schelmenroman, als einen zentralen weltliterarischen Schuldroman, in dem die Ns-Vergangenheit als kollektive Schuld hinterfragt wird. Er lässt viele Fragen offen, so z. B. die Frage nach dem Unterschied zwischen Bosheit, Schuld und Blindheit, nach den Frauenbildern oder der Bedeutung der magischen Bilder im Werk. Dass die Antwort auf diese Fragen nicht einfach ist bzw. dem Text nicht ohne weiteres zu entnehmen ist, demonstriert sie mit der Wiedergabe folgender Leseerfahrung:

Die hässlichste Aussage über Die Blechtrommel habe ich von einem Alten in der Nachbarschaft gehört, als ich ihn dazu befragte. Er sagte, dass es irgendwie so peinlich sei, dass die Phantasie von Grass nicht sehr phantasievoll sei. Ausschweifungen ja, aber keine Überraschungen. Für mich ist Die Blechtrommel wie ein zusammen geklappter Pfannkuchen, der von der Form her phantastisch genug ist, jedoch nicht fest bleibt und deshalb mehr ein burrito ist und auf das Kinn rinnt und über die Brust herunter, so dass es fast beschämend ist. Man kann den Roman hier und da oder vom Anfang bis zum Ende lesen, die Geschichte ändert sich so oder so nicht besonders. ${ }^{64}$

Pulli zieht in ihre Rezension auch das Nachwort von Koskelainen mit ein und wertet es als zwar „verdienstvoll, jedoch sehr biographisch betont“. ${ }^{55}$ Die Kritik an der Person Grass mit seiner Ns-Vergangenheit ist auf die Informationen im Nachwort zurückzuführen und gipfelt in der Verurteilung von Grass als Person, nicht als Autor: „Ich möchte nicht in dieselbe Kneipe mit Grass geraten, falls er mir über sein Leben erzählen würde“66. Was allerdings den Roman anbelangt, so kann sie der Meinung des ,Literaturpapstes‘ Marcel Reich-Ranicki zustimmen, wenn er den Umfang des Romans kritisiert. Der Hinweis auf die Kritik kann im Nachwort zur Übersetzung gelesen werden.

Das Nachwort selbst ist ausschlaggebend für den Literaturkritiker Jari Olavi Hiltunen, dessen Rezension „Romanklassiker über den Krieg eines halberwachsenen Mannes“ im Jahr 2009 in Kiiltomato.net und drei Jahre später in VETUS Et NOVA, einem weiteren Internet-Rezensionsorgan, unverändert veröffentlicht wurde. Hiltunen geht auf die im Nachwort behandelten Stilelemente

\footnotetext{
63 Pulli (2009).

64 Pulli (2009) 55 .

65 Pulli (2009) 55 .

66 Pulli (2009) 54.
} 
und -mittel sowie auf die Themen und Kritik zum Werk ein, was zeigt, dass seine Rezension im Großen und Ganzen auf das Nachwort aufbaut. Er hebt zusätzlich nur zwei weitere Aspekte hervor. Erstens nennt er die gewollte und ungewollte Ironie im Roman, verbunden mit dem Sarkasmus gegenüber der katholischen Kirche, was zur Folge hat, dass die Gespräche Oskars mit Jesus nicht vergleichbar seien mit denen des Don Camillo von Giovanni Guareschi, der als intertextueller Hinweis erwähnt wird. Der zweite Aspekt betrifft die Übersetzungskritik, die ,Konkurrenz der Stimmen', wobei Suominen ihre Position in dieser Neuübersetzung gut vertritt: Ihre Übersetzung sei „klar, anschaulich und dicht; das sprachliche Feuerwerk von Grass hat eine ausgezeichnete finnischsprachige Interpretin bekommen". 67

Die Blog-Beiträge sind in der Zeit von 2009 bis 2014 erschienen. Die Gründe für das Verfassen der Beiträge liegen nicht eindeutig in der Neuübersetzung, denn bis auf den Beitrag von Kemppinen (2009) haben alle die Übersetzung von Peromies aus dem Jahr 1961 als Interpretationsgrundlage. Es darf jedoch davon ausgegangen werden, dass die Neuübersetzung und die Rezensionen in den Medien die Blogger auf das Werk aufmerksam gemacht haben, so dass sie das Werk wieder hervorgeholt und besprochen haben. Bei zwei Bloggern bleiben die Übersetzer unsichtbar, ${ }^{68}$ da es ihnen nur darum geht, Rezensionen über lesenswerte Bücher ${ }^{69}$ bzw. „subjektiv objektive Rezensionen“70 ${ }^{70}$ zu veröffentlichen. Die weiteren Blogger geben als Anlass für ihr Interesse an, entweder sowohl das Buch gelesen als auch die Verfilmung gesehen ${ }^{71}$ oder aber die Stadt Danzig besucht zu haben. ${ }^{72}$

In den Blog-Beiträgen fällt auf, dass nur wenige den Roman so eindeutig werten wie Paalosalo-Jussinmäki, wenn sie „die kräftige Stimme“ im Roman hervorhebt und feststellt: „Ich bin zwischendurch so süchtig danach wie nach Sushi“. ${ }^{73}$ Dagegen stellt sich die Frage nach dem Verstehen eines literarischen Werks in den Vordergrund. So charakterisiert z. B. der Blogger Jokke (2014) die Handlung im Roman als "weder linear noch logisch“, das Buch und seine Hauptfigur als „sonderbar". Sein Fazit lautet, dass die Blechtrommel entweder eine „scheinbar künstlerische, sprachliche Spielerei oder ein zeitloses Meisterwerk" sei. ${ }^{74}$ Auch wenn das Werk ,lesenswert' sei, so bleibt die Wertung

67 Hiltunen (2009).

68 Paalosalo-Jussinmäki (2010); Jokke (2014).

69 Paalasalo-Jussinmäki (2010).

70 Jokke (2014).

71 Tuumailua (2010).

72 Orapihlaja (2010).

73 Paalasalo-Jussinmäki (2010).

74 Jokke (2014). 
doch zwiespältig: Es ist „entweder etwas langweilig Gebackenes oder ein Klassiker, der alle Bereiche des Bewusstseins berührt ${ }^{u}{ }^{75}$ Zudem betont er, dass man nicht wisse, ob man das Buch überhaupt ,verstanden “ habe und inwiefern die Ns-Vergangenheit von Grass das Lesen beeinflussen könne. Der letztgenannte Aspekt wird in den Kommentaren in diesem Blog sehr deutlich hervorgehoben. Dadurch, dass die Blogger die erste Übersetzung als Vorlage benutzt haben, haben sie sich über die Vergangenheit von Grass anderweitig informieren müssen.

Auch der Blogger Tuumailua (2010) ist der Meinung, dass es sich lohne, die Blechtrommel zu lesen, denn es sei ein Roman, der "Gedanken entstehen lässt und unglaublich reich ist". Und trotzdem sei er erzähltechnisch teilweise „nichtssagend“ und beim ersten Lesen nicht zu verstehen. Dieses Unverständnis spiegele sich auch in der Handlung im Film wider, die fragmentarisch und "zersplittert" bleibe. ${ }^{76}$ In den Kommentaren verschiebt sich die Diskussion allgemein auf die unterschiedlichen Kunstformen Buch und Film und auf die Frage, wie die Prioritäten zwischen Buch und Buchverfilmung zu setzen seien.

\section{Zum Schluss}

Die Rezeption der Neuübersetzung der Blechtrommel ist nicht nur durch die auffallend enge Vernetzung der Akteure im Vermittlungsprozess der Literatur gekennzeichnet, sondern spiegelt auch die Unterschiede zwischen den verschiedenen Medien wider. Die Rezeption in den Printmedien konzentriert sich auf folgende drei Punkte: Erstens und vor allem steht der Übersetzungsvergleich im Mittelpunkt, wobei Ausführungen der Übersetzerin in weiteren Dokumenten herangezogen werden. Die anfangs erwähnte ,Polyinterpretabilität' betrifft folglich die Übersetzungen. Zweitens zeigt sich, dass die Bedeutung des Nachworts in der Übersetzung von 2009 von entscheidender Relevanz für die Rezeption ist: Nur aufgrund der im Nachwort gelieferten Informationen können die Rezensenten Die Blechtrommel und ihren Autor in einen soziokulturell-historischen Kontext setzen und darüber in ihren Rezensionen reflektieren. Der dritte Punkt betrifft die Diskussion um die Fiktionalität des Buchs, d. h. das Verhältnis der realen Orte zum fiktionalen Text. Die Entfaltung dieser Diskussion kann ebenfalls auf das Nachwort, auf die bewusste Leselenkung mit Hilfe der Ortsbeschreibung Danzigs von Paavolainen, zurückgeführt werden.

$75 \quad$ Jokke (2014).

76 Tuumailua (2010). 
Eine Polyinterpretabilität ist auch innerhalb der Medien festzustellen. Im Unterschied zu den Printmedien wird in dem sozialen Medium Blog überwiegend das Verstehen eines literarischen Werks besprochen. Dies korreliert zwar mit der Aussage der Übersetzerin Suominen, wonach das Buch unterschiedlich gelesen und interpretiert werden kann, führt aber bei den Bloggern zur Unsicherheit bezüglich der Wertung - lesenswert oder nicht. Der Vergleich der Übersetzungen, der fast immer die Rezeption einer Neuübersetzung dominiert, spielt in den Blogs bis auf eine Ausnahme schon aus dem Grund keine Rolle, weil die Blogger die erste Übersetzung aus dem Jahr 1961 gelesen haben. Die Arbeit des Übersetzers ist einem Amateurblogger nicht so bewusst wie einem professionellen Rezensenten, was dazu führt, dass die Übersetzer in den Blogs unsichtbar bleiben. Auffallend in allen Rezensionen ist, dass die Verleihung des Nobel-Preises an Grass unerwähnt bleibt, auch wenn die Preisverleihung als ein Anlass für die Neuübersetzung angenommen werden kann.

Die Rezeption in Finnland bleibt im Vergleich z. B. zum deutschen Sprachraum recht mager, schon aus dem Grund, dass Grass keinen vergleichbaren Bekanntheits- und Aktualitätsgrad besitzt. Das Buch war schnell vergriffen und es wurden bis zum gegenwärtigen Zeitpunkt keine neuen Auflagen gedruckt, was wiederum auch damit zusammenhängen kann, dass der finnische Markt und die entsprechende Nachfrage relativ klein sind. Die unmittelbaren Reaktionen in der finnischen Öffentlichkeit auf den Tod des Autors lassen jedoch ein wiedererwachendes Interesse erkennen.

\section{Literatur}

Ahola, S., „Kaikki hänen kirjansa ovat myös poliittisia“, in Helsingin Sanomat, April 14, 2015.

Bode, C., Der Roman: Eine Einführung (Tübingen, 2005).

Genette, G., Paratexte. Das Buch vom Beiwerk des Buches (Frankfurt am Main, 2001).

Grass, G., Peltirumpu. Suomentanut Aarno Peromies. Toinen painos (Helsinki, 1967).

Grass, G., Die Blechtrommel (München, 1998).

Grass, G., (2009): Peltirumpu. Suomentanut Oili Suominen. Jälkisana Jukka Koskelainen (Helsinki, 2009).

Hiltunen, J., „Romaaniklassikko keskenkasvuisen miehen sodasta“, July 22, 2009. <http:// www.kiiltomato.net/gunter-grass-peltirumpu/> [Zugriff: 8.11.2014]. Auch 1.11.2012 in: VETUS Et NOVA - Artikkeleita ja arvosteluja uusistaja vanhoista kirjoista. $<$ http://johil tunen.wordpress.com/2012/11/o1/romaaniklassikko-keskenkasvuisen-miehen-sodas ta/>. [Zugriff: 8.11.2014]. 
Ingold, F., „Jede Neuübersetzung ist eine Nachübersetzung. Wie könnten literarische Übersetzer mit der Arbeit ihrer Vorgänger umgehen?“, in Neue Zürcher Zeitung September 29, 2007.

Jokke, <http://joklaaja.blogspot.fi/2014/o2/gunter-grass-peltirumpu.html/> [Zugriff: 8.11.2014].

Kansallisbiografia, <http://www.kansallisbiografia.fi/kb/artikkeli/4818/> [Zugriff: 02.11.2014].

Kemppi, E., „Oskar rummuttaa komeasti Q-teatterissa“, in Turun Sanomat, February 16, 2008.

Kemppinen, „Gdanszsig. 23.4.2009“. <http://kemppinen.blogspot.fi/2009/o4/gdanszsig. html/> [Zugriff: 2.11.2014].

Lähteenmäki, T., „Grassin Peltirummusta uusi suomenkielinen käännös“, in KääntäjäÖversättaren 2009, Heft 4, 5 .

Lassila, P., Otavan historia. Kolmas osa 1941-1975 (Helsinki, 1990).

Launis, K., „Käännökset - kulttuurisen vuorovaikutuksen elinehto“, in Hiidenkivi 4/2012, 27.

Lorenz, S., „Übersetzungstheorie, Übersetzungswissenschaft, Übersetzungsforschung“, in Arnold, H./H. Detering (Hg.), Grundzüge der Literaturwissenschaft (München, 1996), 555-569.

Majander A./J. Petäjä, „Saksa menetti omantuntonsa“, in Helsingin Sanomat, April 14, 2015 .

Mäkelä M., „Peltirumpu“, Februar 21, 2008. <http://www.skenet.fi/artikkeli/o8/o2/pelti rumpu/> [Zugriff: 02.11.2014].

Marttila, H., (2009a) Tausta. Grassin Danzig ja Peltirummun Gdansk. 4.4.20o9. $<$ http:www.hs.online> [Zugriff 8.5.2012].

Marttila, H., (2009b) „Pimeän vuosisadan jeesuslapsi. Oskar Mazeratin rumpu lyö selkeämmin Peltirummun uudessa suomennoksessa“, in Helsingin Sanomat, April 5, 2009 .

Neuhaus, S., Literaturvermittlung (Konstanz, 2009).

Oittinen, R., Kääntäminen uudelleenlukemisena ja uudelleenkirjoittamisena, in Paloposki, O. \& H. Makkonen-Craig (Hg.), Käännöskirjallisuus ja sen kritiikki. (Helsinki, 2000), 265-285.

Orapihlaja, „42. Gdansk ja Helsinki romaaneissa“, 17.4.2010. <http://5555kirjaa.blogspot. fi/2010/04/42-gdansk-ja-helsinki-romaaneissa.html/> [Zugriff: 10.11.2014].

Otava (2014): Otava. Tähtiä kuin Otavassa ... <http://www.otava.fi/yritys > [Zugriff 05.01.2015].

Paalosalo-Jussinmäki, M., „Kuukauden klassikko: Peltirumpu“, April 30, 2010. <http:// www.olivialehti.fi/kirjosieppo/2010/04/3o/kuukauden-klassikko-peltirumpu/> [Zugriff: 15.9.2012].

Paavolainen, O., Lähtö ja loitsu. Kirja suuresta levottomuudesta (Helsinki, 1978). 
Pulli, T., „Veijari ja Musta Köksä“, in Parnasso 5/2009, 54-55.

Pöckl, W., „Zwischen Zufall und Notwendigkeit: Neuübersetzungen“, in Internationales Archiv für Sozialgeschichte der deutschen Literatur, 29 (2004), 2, 200-210.

Pöckl, W., „Wirkungsäquivalenz“, in Neuhaus, S. und O. Ruf (Hg.), Perspektiven der Literaturvermittlung (Innsbruck/Wien, 2011), 38-50.

Saari, J., Oskarin uusi Peltirumpu: näkökulmia uudelleenkääntämiseen. Unveröffentlichte Magisterarbeit (Turku, 2011).

Saarikoski, P., „Cinerama“, in Parnasso 5/1961, 257-258.

Särö, M.: Teatteriarvio: Q-teatterin Peltirumpu häkellyttää barokkisuudellaan. <http://www.uusisuomi.fi/kulttuuri/14932-q-teatterin-peltirumpu-hakellyttaabarokkisuudellaan/> [Zugriff: 30.10.2014].

Stolze, R., „Sprachphilosophie (Hermeneutik)“, in Snell-Hornby, M., H. Hönig, P. Kußmaul, P. Schmitt (Hg.), Handbuch Translation (Tübingen, 1998), 115-119.

Suomen kirjataiteen komitea (2009) = <http://www.kauneimmatkirjat.fi/arkisto/2009/ kaunokirjallisuus/\#op> [Zugriff: 8.11.2014].

Tiilikainen, T., „Günter Grassin Peltirumpu heräsi uudelleen henkiin“, in <http://suomen kuvalehti.fi/jutut/kulttuuri/gunter-grassin-peltirumpu-herasi-uudelleen-henkiin/> [Zugriff: 8.11.2014].

Tiittula. L., „Finnische Neuübersetzungen deutschsprachiger Literatur“, in trans-kom (2013), 1, 140-170.

Toivanen, T., „Ennen muuta kääntäjän pitää rakastaa äidinkieltään“. Haastattelussa kääntäjä Oili Suominen, in AVAIN 2006/1, 48-57.

Tuumailua, „Peltirummun päristessä“, 4. January 21, 2010. < http://tuima.vuodatus.net/ lue/2010/o1/peltirummun-paristessa-4/> [Zugriff: 4.11.2014.]. 